\title{
Estimación del riesgo vascular global a través de un puntaje europeo y una ergometría
}

\begin{abstract}
Objetivo
Determinar en individuos asintomáticos la validez del puntaje de Framingham (PF) desarrollado en EEUU para predecir la mortalidad por todas las causas y del puntaje SCORE (PS) Europa, para predecir la mortalidad cardiovascular; 2) Determinar el valor pronóstico de la ergometría en pacientes a quienes ya se ha calculado su riesgo basal con alguno de los dos instrumentos mencionados previamente.
\end{abstract}

\section{Diseño}

Cohorte prospectiva.

\section{Pacientes}

Un total de 3554 adultos asintomáticos de 50 a 75 años que fueron sometidos a una ergometría como parte de un programa de salud entre octubre de 1990 y diciembre de 2002, y fueron seguidos un promedio de ocho años.

\section{Medición de los resultados principales}

1) Riesgo Global según el PF y el PS; 2) Anormalidades de la ergometría recolectadas en forma prospectiva (mala condición física, recuperación anormal del ritmo cardíaco, ectopía ventricular y anormalidades del segmento ST). El punto final primario fue la mortalidad por todas las causas.

\section{Resultados}

Hubo 114 muertes. De acuerdo a sus características operativas resumidas en el índice $\mathrm{C}$ de la curva $\mathrm{ROC}^{*}$ y a los Criterios de
Información de Akaike*, el PS fue superior al PF en la estimación del riesgo global de mortalidad. Luego del análisis multivariable, fueron predictores independientes de muerte los que se describen en el cuadro 1.

Cuadro 1: predictores independientes de muerte.

\begin{tabular}{|c|c|c|}
\hline & & $\begin{array}{l}\text { Aumento de la mortalidad } \\
\text { global confirmado en forma } \\
\text { prospectiva (RR, IC95\%) }\end{array}$ \\
\hline \multicolumn{2}{|c|}{$\begin{array}{l}\text { Aumento de } 1 \% \text { en el riesgo absoluto predicho } \\
\text { por el puntaje europeo SCORE }\end{array}$} & $1,07(1,04$ a 1,09$)$ \\
\hline \multirow[t]{2}{*}{ Ergométricas } & Mala capacidad funcional & 2,$95 ;(1,98$ a 4,39$)$ \\
\hline & $\begin{array}{l}\text { Recuperación anormal del } \\
\text { ritmo cardíaco }\end{array}$ & 1,$59 ;(1,04$ a 2,41$)$ \\
\hline
\end{tabular}

La depresión del segmento ST no predijo la mortalidad. Entre los pacientes ubicados en el tercilo más alto del PS, un resultado anormal de la ergometría definido como mala capacidad funcional o recuperación anormal del ritmo cardíaco, identificó a un grupo con un riesgo de $1 \%$ de muerte anual.

\section{Conclusión}

La combinación entre el PS y la ergometría puede ser útil para estratificar el riesgo cardiovascular en individuos asintomáticos.

Fuente de financiamiento: Nacional Heart, Lung and Blood Institute.

\section{Comentario}

El desarrollo de puntajes para evaluar el riesgo de eventos cardiovasculares en sujetos asintomáticos ha permitido identificar grupos con riesgo elevado (mayor a $20 \%$ a diez años) en quienes las intervenciones preventivas tienen gran utilidad. Ver figura 1.

Sin embargo, existen áreas imprecisas en la estratificación en que las estimaciones no pueden determinar con exactitud el riesgo que guíe las intervenciones, como en aquellos sujetos con un riesgo a diez años entre 10 y $20 \%$. Con el objetivo de mejorar la precisión estas estimaciones, diversas variables están siendo estudiadas. Algunas de ellas son mediciones anatómicas como el índice tobillo brazo, o el espesor de la media- íntima carotídea; y otros, marcadores bioquímicos como la proteina $\mathrm{C}$ reactiva o pruebas funcionales de ejercicio como la del artículo que comentamos ${ }^{1}$.

El fundamento de la utilización de las pruebas funcionales radica en que reducciones en el tono vagal aumentan el riesgo de muerte súbita, independientemente del compromiso de las arterias coronarias ${ }^{2}$. Debe tenerse presente que la solicitud de una prueba funcional en un sujeto asintomático tiene otra interpretación a la realizada en un sujeto con dolor torácico. la primera tiene un criterio de rastreo, mientras que la segunda es una búsqueda de caso aplicando un criterio bayesiano ${ }^{3}$. No debe confundirse este criterio, ya que las variables que surgen son diferentes; por ejemplo, la depresión del segmento ST, fundamental para el diagnóstico y pronóstico en sujetos sintomáticos no resulta predictora en estudios de sujetos asintomáticos ${ }^{4}$

A pesar de que en el presente estudio las pruebas funcionales mejoraron la capacidad de predicción, las sociedades científicas no adoptaron la prueba de ejercicio como práctica rutinaria de rastreo ${ }^{4,5}$, ya que si bien es claro que el riesgo en distintas cohortes parece ser mayor, no se sabe si la modificación, por ejemplo a través de un programa de ejercicio, de las variables que aparecieron asociadas a un peor pronóstico (ej. mala capacidad funcional, extrasistolia ventricular durante la recuperación) modificaría este riesgo ${ }^{6}$.

Con respecto a la comparación entre los puntajes de riesgo, el de Framingham proviene de una muestra pequeña seguida por muchos años en un lugar especifico y determinado de los Estados Unidos, y si bien, ha sido validado en algunas poblaciones, no lo ha sido en otras, donde su capacidad de predicción ha sido cuestio-nada.

EI SCORE es un puntaje desarrollado por la Sociedad Europea de Cardiología, utiliza las mismas variables que el de Framingham y, se realizó en distintos países. Por este último motivo incluyó coeficientes para ajustar el riesgo basal de la población en estudio ${ }^{6}$. En el presente estudio y comparado con el de Framingham, el puntaje SCORE aparece como un mejor predictor, lo que resulta interesante ya que el estudio se realizó en una muestra de la población norteamericana. Una de las explicaciones posibles sería que el puntaje Framingham mide infarto no fatal y muertes cardiovasculares, mientras que el SCORE se desarrolló midiendo solo esta última variable. Además, el registro de eventos cadiovasculares no fatales tiene mucha mayor variabilidad y es más dificultoso fuera del ámbito experimental como el utilizado para el desarrollo del puntaje Framingham.

Se necesitarán comparaciones de distintas cohortes por un tiempo más prolongado para poder determinar con mayor exactitud qué puntaje predice mejor el riesgo cardiovascular. Por un lado podemos concluir que los sujetos cuyo puntaje se encuentra en el tercilo* superior de la distribución, ya están por si mismo en una categoría de riesgo mucho mayor y las pruebas de ejercicio no añaden por ahora información que condicione una intervención especifica distinta al control más o menos agresivo de los factores de riesgo. Por otro lado, no hay todavía evidencia proveniente de estudios aleatorizados que demuestre que conocer el riesgo de un paciente individual disminuya la mortalidad cardiovascular, siendo la prueba de ejercicio una posible herramienta de rastreo.

\section{Conclusiones de los comentadores:}

EI puntaje SCORE podría ser una herramienta de rastreo con mayor validez externa que el puntaje de Framingham, mientras que la prueba de ejercicio deberá seguir siendo estudiadas para evaluar si puede mejorar la estimación del riesgo cardiovascular, utilizada en forma secuencial a los puntajes de riesgo. 
Figura 1: calculadora en castellano para la estimación del riesgo cardiovascular global disponible en Internet.

Cálculo de Riesgo Cardíaco a los 10 años (puntaje de Rieso de Framingham, según ATPIII)

\section{Factores de Riesgo}

\begin{tabular}{|c|c|c|c|c|}
\hline Sexo & () $\mathrm{M} O$ & & \multirow{5}{*}{$\begin{array}{l}\text { Presenta alguno de las siguientes enfermedades: } \\
\text { - Enfermedad coronaria comprobada } \\
\text { - Enfermedad carotidia sintomática } \\
\text { - Enfermedad arterial periferica } \\
\text { - Aneurisma aorta abdominal } \\
\text { - Diabetes }\end{array}$} & \multirow{5}{*}{$\square$} \\
\hline Edad & $50-54$ & $\nabla$ & & \\
\hline Tabaquista & \multicolumn{2}{|c|}{ ○ $\mathrm{Si} \bigcirc$ No } & & \\
\hline TA sistólica & \multicolumn{2}{|c|}{\begin{tabular}{|l|l|}
$120-129$ & $\nabla$ \\
\end{tabular}} & & \\
\hline $\begin{array}{l}\text { TA Bajo } \\
\text { Tratamiento? }\end{array}$ & \multicolumn{2}{|c|}{ O si $\bigcirc$ No } & & \\
\hline $\begin{array}{l}\text { Colestrol } \\
\text { Total }\end{array}$ & $160-199$ & $\nabla$ & \multirow{3}{*}{$\begin{array}{l}\text { Historia familiar de enfermedad coronaria prematura ( en familiar } \\
\text { de primer grado masculino }<55 \text { años, femenino }<65 \text { años.) }\end{array}$} & \multirow{3}{*}{$\square$} \\
\hline HDL & $40-49$ & 7 & & \\
\hline \multirow[t]{2}{*}{ LDL } & 130-159 & $\nabla$ & & \\
\hline & $\begin{array}{c}<100 \\
100-129 \\
130-159 \\
160-189 \\
>=190\end{array}$ & & Calcular Riesgo & \\
\hline
\end{tabular}

De los Ríos E, López Osornio A. Calculadora clínica para la evaluación del Riesgo Cardiovascular a diez años. Basada en el Executive Summary of the Third Report of the National Cholesterol Education Program (NCEP) Expert Panel on Detection, Evaluation, and Treatment of High Blood Cholesterol in Adults (Adult Treatment Panel III), JAMA. 2001;285:2486-2497. Disponible libremente en Internet: http://www.foroaps.org/hitalba-desarrollo-calculadoras.php?cod_producto=1531 (ultima entrada: agosto 2005).

Augusto Granel y Sergio Terrasa [ Unidad de Medicina Familiar y Preventiva, Hospital Italiano de Buenos Aires. ]

Terrasa S, Granel A. Estimación del riesgo vascular global a través de un Puntaje europeo y una ergometría. Evid. actual. práct. ambul. 2005;8:108109.Comentado de: Aktas M, Ozduran V, Pothier C, Lang R, Lauer M. Global risk scores and exercise testing for predicting all-cause mortality in a preventive medicine program. PMID: 15383517. JAMA. 2004 Sep 22;292(12):1462-8.

\section{Referencias}

1. Green land P, Smith SC, Grundy SM. Improving coronary risk assesment i asymptomatic people: role of traditional risk factors and non invasive cardiovascular tests. Circulation 2001;104:1863.1867

2. Vevierkananthan $\mathrm{DB}$, Blackstone $\mathrm{EH}$, Pottier $\mathrm{CB}$, et al. heart rate recovery after exercise is a predictor of mortality independent of angiographyc severity of coronary disease. J Am Coll Cardiol 2003; $42: 831-838$

3. Green lan P, Graziano M. Selecting asymptomatic patients for coronary computed tomography or electrocardiographyc exercise testing. N Engl J Med .2003; 349:465-473

4. Fowler-Brown A, Pignone M, Pletcher M et al. Excercise tolerance testing to screen for coronary heart disease: a systematic review for the technical support for the US preentive services Task Force. Ann Intern Med. 2004;140:w9-w24

5. Gibbons RJ, Balady GJ, Bricker JT et al. ACC/AHA 2002 guideline upate for execrcise testing : summary article. Circulation 2002;106;1883-1892

6. Conroy RM, Pyorala K. Fitagerald AO, et al. Estimation of ten year risk of fatal cardiovascular disease in Europe: the SCORE proyect. Eur Heart J. 2003:24:987-1003 